\title{
Excipients: The Real Players behind Robust Formulation
}

\author{
Hitesh Chavda*, Suresh Kumar Neerumalla, Axay Patel, Sanjay Poptani \\ Department of Formulation and Development, R\&D Centre, Gowrie Research Pvt Ltd (B\&S Group), Vadodara - 391740, Gujarat, INDIA.
}

The backbone of any successful robust pharmaceutical product is built on their excipients. Excipients show a vital role in the dosage form design. Excipients do not have therapeutic activity, however, they influence the therapeutic activity of active pharmaceutical ingredient (API) through formulation characteristics. Please note inactive ingredients are clearly not reliably inert in their biological activity and thus should not be listed as such. A more suitable and concise term is excipient.

A bad or wrong excipient selection can even lead to several issues. In Australia, in the late 1960s, epileptic patients who were taking phenytoin capsules experienced intoxications due to the replacement of diluent, calcium sulphate with lactose in the capsule formulation. This substitution, considered to be harmless, give rise to in an increase of the mean serum concentration of phenytoin by a factor of 4.5, which is large for a narrow therapeutic index drug such as phenytoin. This happens due to the solubility of excipients in water. Calcium sulphate (dehydrate) is very slightly soluble in water whereas as lactose is freely soluble in water. Therefore, in the original capsule formulation, calcium sulphate performed as a matrix former and prolonged the release of the phenytoin, whereas lactose triggered an immediate and considerable release of phenytoin beyond the toxic threshold. ${ }^{1-2}$ Misperception between excipients can also have a serious outcome. Toxic cough syrup case refers to a 2007 scandal in which Panamanian pharmaceutical manufacturers formulated cough syrup using diethylene glycol which they understood to be glycerin. Diethylene glycol is a cheaper alternative to glycerin for industrial applications, however, it is nephrotoxic. As reported by The New York Times 365 deaths from the poison, 100 of which have been confirmed so far. ${ }^{3}$ In India and Bangladesh, in 1990-1992 paracetamol syrup in which diethylene glycol was substituted for propylene glycol caused 236 deaths. ${ }^{4}$ In USA, 71 adults and 34 children died in 1937 after taking a drug "Elixir Sulfanilamide" to treat a variety of ailments, from gonorrhea to sore throat. The investigators from the US Food and Drug Administration (FDA) identified the solvent, diethylene glycol, used in formulation as the killer. ${ }^{5}$ In pediatric population the presence of benzalkonium chloride in antihistaminic drugs leads to bronchospasms, aspartame (sweetener) leads to headaches and seizures. The presence of propylene glycol in neonates leads to hyper-osmolality and lactic acidosis. ${ }^{6}$ Benzyl alcohol toxicity in neonates was reported in 1980. A gasping syndrome was reported in premature infants. ${ }^{7}$ At this time in the United States, bacteriostatic water and sodium chloride injections contained $0.9 \%$ benzyl alcohol. The infants received injections from flushes and/or medicines which were reconstituted with the water and sodium chloride. The pathways involved in the metabolism, benzyl alcohol is oxidized to benzoic acid and then in the liver it is conjugated with glycine and finally excreted as hippuric acid in the urine. In premature babies this metabolism pathway is immature, and as a result these infants accumulated benzyl alcohol and leads to toxicity. ${ }^{7}$

Excipients selection for a drug product is a key step in drug development. The selection
Submission Date: 13-03-2018; Revision Date: 05-06-2018; Accepted Date: 14-08-2018

DOI: 10.5530/ijper.53.1.2 Correspondence:

Dr. Hitesh Chavda Department of Formulation and Development, R\&D Centre,

Gowrie Research Pvt Ltd (B\&S Group), Vadodara 391740, Gujarat, INDIA. Phone: +919427472354 E-mail: hitcvd@gmail.com

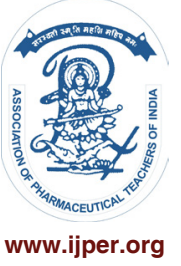


of excipients for the particular pharmaceutical product is a very critical step and it depends upon the API, route of administration, dosage form and other factors. Definitely, the properties like bioavailability and stability of the ultimate dosage form are greatly dependent on the excipients selected, their concentration and interaction with both the API and each other. A thorough knowledge of excipients is crucial for scientists. Furthermore, the evolution of novel forms of drug delivery has brought about in an increase in the total of the excipients being used. Scientists must study stability and compatibility issue, pharmacokinetic aspects, permeation characteristics, drug delivery platform, segmental absorption performance, intellectual property concerns etc. while choosing an excipient for development of robust formulation. APIs cannot be administrated as such; therefore they are formulated in suitable dosage forms for the several reasons. These reasons may be dose accuracy and consistency, patient compliance, bioavailability enhancement, aesthetics and side effects reduction. Excipients are the essential necessities to these reforms allowing formulation scientists to accomplish their set objectives.

A carefully-selected excipient may lessen manufacturing costs, may amplify patient experience, etc. An excipient must suit the proposed dosage form, establish organoleptic properties, obey pharmacopeial regulations, easily available and work excellently. It should work well with equipment. Determining factors may include the amount of drug needed, the proposed use of the drug, prevailing environmental conditions, excipient potential toxicity and its origin. ${ }^{8}$

There are diverse types of excipients and their requirements. Excipients may be a single chemical entity (include organic and inorganic acids, their salts, sugars, alcohols, etc.), may have experienced physical treatments (like micronization), may have chemically converted (like modified starch) or mixtures of chemically related components (like polyol esters). Mixed excipients are preparations that are ready-for-use, like ready-made coating materials. Commonly used excipients are listed in Figure 1.

The quantity of excipient to be used in the formulation may be referred from Handbook of Pharmaceutical excipients, ${ }^{9}$ Joint $\mathrm{FAO} / \mathrm{WHO}$ Expert Committee on Food Additives (JECFA), ${ }^{10}$ Inactive Ingredients Database (IID) ${ }^{11}$ etc. Information on inactive ingredients present in FDA-approved drug products is provided by the Inactive Ingredient Database by FDA which can be used by industry as a support in the development of drug products. For different drug development purposes, once an inactive ingredient has appeared in an approved
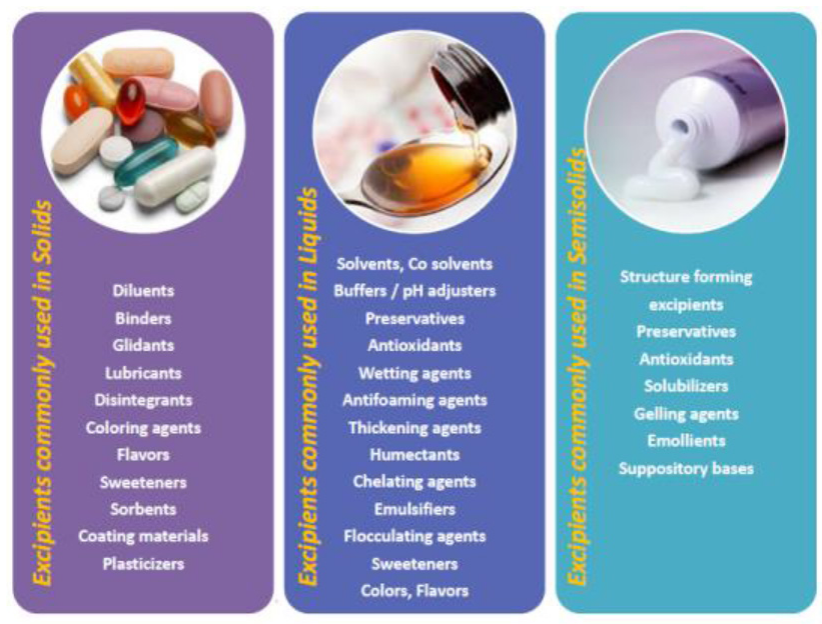

Figure 1: Commonly used excipients for solid, liquid and semisolid products.

drug product database for a particular route of administration, the inactive ingredient is not considered new and may necessitate a less extensive review the next time it is included in a new drug product. According to $21 \mathrm{CFR}$ 210.3(b)(8), an inactive ingredient is any component of a drug product other than the active ingredient. ${ }^{12}$ Novel excipient is a material or composition that has not been previously used in an approved drug product in the US (i.e. not listed in FDA IID) or that has been previously used in an approved drug product but which it is desired to employ for a new route of administration, or a higher level of use that has been previously listed in the IID. ${ }^{13} \mathrm{~A}$ novel excipient can also be defined as a new co-processed excipient made from two or more previously approved excipients. Generally Recognized As Safe (GRAS) excipient is any substance that is intentionally added to food is a food additive, that is subject to premarket review and approval by FDA, unless the substance is generally recognized, among qualified experts, as having been adequately shown to be safe under the conditions of its intended use or unless the use of the substance is otherwise excepted from the definition of a food additive. ${ }^{14}$ The International Pharmaceutical Excipients Council (IPEC), a pharmaceutical regulatory non-profit, develops, implements, and promotes global use of appropriate quality, safety, and functionality standards for pharmaceutical excipients and excipient delivery systems. IPEC functions as a primary international resource on excipients for its members, governments, and public audiences. ${ }^{15} \mathrm{~A}$ group of industry experts from European Fine Chemical Group (EFCG), IPEC Europe, IPEC Americas, European Association of Chemical Distributors (FECC), and Pharmaceutical Quality Group (PQG) have worked 
together since 2008 on the development of a certification scheme for excipients suppliers now known as EXCIPACT $^{\mathrm{TM}}$. The EXCIPACT ${ }^{\mathrm{TM}}$ scheme provides for an independent certification of manufacturers and suppliers of excipients as a means of ensuring patient safety, improving assurance of supplier quality, while minimizing the overall supply chain costs. ${ }^{16}$

Implementation of Quality-by-Design (QbD) for a drug product drives the need for $\mathrm{QbD}$ approach for the excipient. Presently there is no specific industry for pharmaceutical excipients. The majority of pharmaceutical excipients are generally provided by chemical or food industries. Specifications are generally not provided by the pharmaceutical market. QbD consideration may encourage pharmaceutical manufacturers choices for excipient selection to choose excipients which are manufactured under well-defined controls, well characterized for a variety of functionalities, possess good batch uniformity, high-quality grade specifically designed for the pharmaceutical purpose and are supplied by authenticated manufacturers. ${ }^{17}$

As per report "Global Pharmaceutical Excipients Market Growth, Trends and Forecasts (2017 - 2022)" published by MarketResearch.com the global market of pharmaceutical excipients is anticipated to reach USD 9.3 billion by the end of 2020, rising at a CAGR of about $7.2 \%$ from 2014 to $2021 .{ }^{18}$

It is important that scientists ensure that they have fully investigated excipients to be used for the development of the most robust formulation, as excipients are the backbone and real players.

\section{REFERENCES}

1. Bochner F, Hooper WD, Tyrer JH, Eadie MJ. Factors involved in an outbreak of phenytoin intoxication. Journal of the Neurologic Sciences. 1972;16(4):481-7.

2. Inactive Ingredient Search for Approved Drug Products. U.S. Food and Drug Administration. 2018 [cited 2018 Apr 17]. Available from: URL: https://www. accessdata.fda.gov/scripts/cder/iig/index.cfm

3. Bogdanich W, Hooker J. From China to Panama, a Trail of Poisoned Medicine. The New York Times. 2007 [cited 2018 Apr 17]. Available from: URL: http://www.nytimes.com/2007/05/06/world/americas/06poison.html
4. Taylor P. IPEC Europe. Excipients Insight. 2009 [cited 2018 Apr 17]. Available from: URL: http://ipec-europe.org/UPLOADS/Excipients_Insight_Mar09_ Final.pdf

5. Akst J. The Elixir Tragedy, 1937. The Scientist. 2013 [cited 2018 Jun 20]. Available from: URL: https://www.the-scientist.com/?articles.view/articleNo/ 35714/title/The-Elixir-Tragedy--1937/

6. Chatterjee P, Yochana S, Yu M, Alvi M, Varenya S. Pharmaceutical excipients and pediatric formulations. Chimica oggi/Chemistry Today. 2012;30(5):56-61.

7. Gershanik J, Boecler B, Ensley H, McCloskey S, George W. The gasping syndrome and benzyl alcohol poisoning. The New England Journal of Medicine. 1982;307(22):1384-8.

8. Auerbach M. Pharmaceutical Excipients. American Pharmaceutical Review [cited 2018 Apr 17] Available from: URL: http://www. americanpharmaceuticalreview.com/25335-Pharmaceutical-Raw-Materialsand-APIs/25283-Pharmaceutical-Excipients/

9. Sheskey PJ, Rowe RC. Handbook of Pharmaceutical excipients. $7^{\text {th }}$ ed. London: The Pharmaceutical Press. 2009.

10. Evaluations of the Joint FAO/WHO Expert Committee on Food Additives (JECFA). World Health Organization [cited 2018 Apr 17]. Available from: URL: http://apps.who.int/food-additives-contaminants-jecfa-database/search.aspx

11. Inactive Ingredient Search for Approved Drug Products. U.S. Food and Drug Administration. 2018 Mar 5 [cited 2018 Apr 17]. Available from: URL: https:// www.accessdata.fda.gov/scripts/cder/iig/index.cfm

12. Inactive Ingredient Search for Approved Drug Products: Frequently Asked Questions. U.S. Food and Drug Administration. 2017 [cited 2018 Apr 17]. Available from: URL: https://www.fda.gov/Drugs/InformationOnDrugs/ ucm080123.htm\#what\%20is\%20inactive\%20ing

13. IPEC Americas and IQ Consortium - FDA meeting to discuss Novel Excipients Initiative. U.S. Food and Drug Administration. 2015 [cited 2018 Apr 17]. Available from: URL: https://www.fda.gov/downloads/AboutFDA/Centers Offices/OfficeofMedicalProductsandTobacco/CDER/UCM461274.pdf

14. Frequently Asked Questions About GRAS for Substances Intended for Use in Human or Animal Food: Guidance for Industry. U.S. Food and Drug Administration. 2016 Oct [cited 2018 Apr 17]. Available from: URL: https://www.fda.gov/downloads/Food/GuidanceRegulation/Guidance DocumentsRegulatoryInformation/UCM525233.pdf

15. International Pharmaceutical Excipients Council of the Americas [cited 2018 Apr 17]. Available from: URL: http://ipecamericas.org/

16. EXCIPACT. Certification Standards for Pharmaceutical Excipients. $2011 \mathrm{Mar}$ 1 [cited 2018 Apr 17]. Available from: URL: http://ipecamericas.org/sites/ default/files/ExcipactVersion2.pdf

17. Schoneker D. The Impact of Excipient Variability on QbD. The Need for Good Qualification Processes - IPEC America's Perspective [cited 2018 Apr 17]. Available from: URL: http://www.ich.org/fileadmin/Public_Web_Site/Training/ GCG_-_Endorsed_Training_Events/APEC_LSIF_JCCT_workshop_ Beijing_China_Dec_08/Day_3/Impact_of_Excipient.pdf

18. Pharmaceuticals Market Research Reports and Industry Analysis [cited 2018 Apr 17]. Available from: URL: https://www.marketresearch.com/LifeSciences-c1594/Pharmaceuticals-c89/

Cite this article: Chavda H, Neerumalla S, Patel A, Poptani S. Excipients: The Real Players behind Robust Formulation. Indian J of Pharmaceutical Education and Research. 2019;53(1):05-07. 